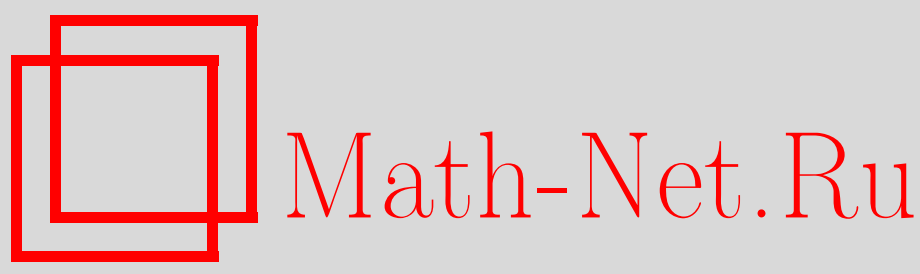

М. Б. Карманова, Минимальные поверхности-графики на произвольных двуступенчатых группах Карно, Изв. вузов. Матем., 2019, номер 5, 15-29

DOI: https://doi.org/10.26907/0021-3446-2019-5-15-29

Использование Общероссийского математического портала Math-Net.Ru подразумевает, что вы прочитали и согласны с пользовательским соглашением

http://www . mathnet.ru/rus/agreement

Параметры загрузки:

IP : 35.174 .16 .151

26 апреля 2023 г., 08:05:24 
Известия вузов. Математика

2019, № 5, c. 15-29 https://kpfu.ru/science/nauchnye-izdaniya/ivrm

e-mail: izvuz.matem@kpfu.ru

\title{
М.Б. КАРМАHOBA
}

\section{МИНИМАЛЬНЫЕ ПОВЕРХНОСТИ-ГРАФИКИ НА ПРОИЗВОЛЬНЫХ ДВУСТУПЕНЧАТЫХ ГРУППАХ КАРНО}

\begin{abstract}
Аннотация. Установлены базовые свойства минимальных поверхностей-графиков, построенных по классу отображений двуступенчатых групп Карно. Методы исследования включают решение характерного для изучаемого случая вопроса о корректности постановки задачи. Основной результат о необходимых условиях минимальности сформулирован в терминах субриманова аналога средней кривизны.
\end{abstract}

Ключевые слова: двуступенчатая группа Карно, отображение-график, минимальная поверхность, средняя кривизна.

УДК: $517.2: 517.4: 514.7$

DOI: $10.26907 / 0021-3446-2019-5-15-29$

\section{ВВЕДЕНИЕ}

Проведенные в статье исследования открывают основные свойства экстремальных поверхностей на неголономных структурах произвольных размерностей. Их известными примерами являются группы Карно. Такие структуры, как и их обобщения - пространства Карно-Каратеодори, применяются при моделировании и решении разнообразных теоретических и прикладных задач (см., например, комментарии и литературные источники в [1]). Цель настоящей работы состоит в рассмотрении отображений-графиков, построенных по классу отображений двуступенчатых групп Карно, и описании соответствующих минимальных поверхностей. Здесь и далее под термином "минимальная поверхность" будем понимать поверхность с минимальной площадью (подразумевается, что решение соответствующей краевой задачи существует). Специфика изучаемых структур и особенности соответствующих отображений порождают вопросы, не возникающие в евклидовом анализе или на неголономных структурах малых размерностей. В частности, в отличие от [2], [3], вопросы о корректности определения приращения функционала возникают уже при описании свойств горизонтального подпространства прообраза. Дело в том, что, как и в классическом анализе, вывод уравнений экстремальных поверхностей основан на дифференцировании функционала площади, который зависит от задающего поверхность отображения. Но если в евклидовом анализе можем заменить исходное отображение $\varphi$ на измененное $\varphi+\varepsilon \psi$ того же класса, то на неголономных структурах так уже сделать не можем в силу зависимости координат степени два и выше от горизонтальных. Кроме того, когда горизонтальных полей более двух, то в силу такой зависимости возникает вопрос о свойствах их

Поступила в редакцию 26.03.2018, после доработки 17.07.2018. Принята к публикации 26.09.2018

Благодарности. Работа выполнена при поддержке программы фундаментальных научных исследований СО РАН № 1.1.2, проект № 0314-2016-0006. 
коммутаторов и их взаимосвязи с векторными полями высоких степеней, так как от таких соотношений напрямую зависит класс, в котором будут рассматриваться поверхности на предмет экстремальности их площади. Помимо этого, как и в [2], [3], во-первых, функционал площади будет меняться неодинаково для координат, стоящих при полях разных степеней, что также добавляет задаче сложность и специфику, и, во-вторых, для неголономных отображений понятие "экстремум площади" и "экстремум значений функционала площади" различны, так как во втором случае функционал может принимать некоторое значение, но существование самого отображения, определяющего поверхность с данной площадью, не обязательно (в силу того, что соответствующая задача в частных производных не всегда разрешима). Это различие является характерным для многомерного случая, когда график определяется отображением, а не функцией (ср., например, [4]).

В первом разделе приведем базовые определения, во втором опишем условия, при которых постановка задачи о минимальных поверхностях корректна, а в третьем сформулируем и решим задачу о минимальных поверхностях.

\section{1. ДВУСТУПЕНЧАТЫЕ ГРУППЫ КАРНО: осНОвНЫЕ ОПРЕДЕЛЕНИЯ}

Определение 1 (например, [5]). Двуступенчатой группой Карно называется связная односвязная стратифицированная группа Ли $\mathbb{G}$, алгебра Ли $V$ которой представляется в виде прямой суммы

$$
V=V_{1} \oplus V_{2}, \quad\left[V_{1}, V_{1}\right]=V_{2}, \quad\left[V_{1}, V_{2}\right]=\{0\} .
$$

Определение 2. Если в (1) условие $\left[V_{1}, V_{1}\right]=V_{2}$ заменить на $\left[V_{1}, V_{1}\right] \subset V_{2}$ и $\left[V_{2}, V_{2}\right]=\{0\}$, то $\mathbb{G}$ называется двуступенчатой нильпотентной градуированной группой (Ли).

Из определений следует, что группа Карно является частным случаем нильпотентной градуированной группы.

Определение 3. Пусть $N$ - топологическая размерность группы $\mathbb{G}$, и $X_{1}, X_{2}, \ldots, X_{N}-$ левоинвариантные векторные поля на $\mathbb{G}$, образующие базис алгебры Ли $V$, причем

$$
\begin{aligned}
& X_{1}, \ldots, X_{\operatorname{dim} V_{1}} \text { - базис } V_{1}, \\
& X_{\operatorname{dim} V_{1}+1}, \ldots, X_{N} \text { - базис } V_{2} .
\end{aligned}
$$

Здесь символ $\operatorname{dim} V_{1}$ означает размерность $V_{1}$. Если $X_{j} \in V_{k}, k=1,2$, то число $k$ называется степенъю поля $X_{j}$ и обозначается символом $\operatorname{deg} X_{j}, j=1, \ldots, N$. Векторные поля степени 1 далее будем называть горизонтальными. В рассматриваемом случае глубина группы $\mathbb{G}$ равна двум.

Замечание 1. Базис выбран таким образом, что его поля имеют либо степень один, либо степень два на всей группе $\mathbb{G}$.

Пусть символ $\mathbf{0}-$ единица группы $\mathbb{G}$.

Из формулы Бейкера-Кэмпбелла-Хаусдорфа и условия левоинвариантности базисных полей выводятся следующие выражения для групповой операции на $\mathbb{G}$. Если $x=\exp \left(\sum_{j=1}^{N} x_{j} X_{j}\right)(\mathbf{0}), y=\exp \left(\sum_{j=1}^{N} y_{j} X_{j}\right)(\mathbf{0})$, то $x \cdot y=z=\exp \left(\sum_{j=1}^{N} z_{j} X_{j}\right)(\mathbf{0})$, где

$$
\begin{array}{ll}
z_{j}=x_{j}+y_{j} & \text { для } \operatorname{deg} X_{j}=1, \\
z_{j}=x_{j}+y_{j}+\sum_{\substack{\mu>0, \beta>0 \\
\mu, \beta \leq \operatorname{dim} V_{1}}} F_{\mu, \beta}^{j} x^{\mu} y^{\beta} \quad \text { для } \operatorname{deg} X_{j}=2 .
\end{array}
$$


Здесь умножение $x \cdot y$ понимается в следующем смысле. Сначала движение идет вдоль интегральной линии векторного поля $\sum_{j=1}^{N} x_{j} X_{j}$ с началом в $\mathbf{0}$ до точки $x$, а затем - вдоль интегральной линии векторного поля $\sum_{j=1}^{N} y_{j} X_{j}$ с началом в $x$. Таким образом, интегральная линия поля $\sum_{j=1}^{N} z_{j} X_{j}$ соединяет точки 0 и $z=\exp \left(\sum_{j=1}^{N} y_{j} X_{j}\right)(x)$.

Определение 4. Константы $\left\{F_{\mu, \beta}^{j}\right\}_{j, \mu, \beta}$ называются структурными константами груп$n$ sы $\mathbb{G}$.

На группах Карно часто используется метрика Карно-Каратеодори $d_{c c}$, согласованная с неголономной структурой (например, [6]). Однако до сих пор остаются открытыми вопросы о строении ее шаров, кроме того, ее не всегда возможно использовать на нильпотентных градуированных группах, поэтому в таких случаях применяется квазиметрика $d_{\infty}$, которая на группах Карно локально билипшицево эквивалентна $d_{c c}$ : если $w=\exp \left(\sum_{i=1}^{N} w_{i} X_{i}\right)(v)$, то $d_{\infty}(v, w)=\max _{i=1, \ldots, N}\left\{\left|w_{i}\right|^{\frac{1}{\operatorname{deg} X_{i}}}\right\}$. Мы же будем использовать следующую величину, локально билипшицево эквивалентную $d_{\infty}$ (см. далее замечание 2 ).

Определение 5 . Пусть $\mathbb{G}$ - нильпотентная градуированная группа топологической размерности $N$, и $w=\exp \left(\sum_{i=1}^{N} w_{i} X_{i}\right)(v)$. Определим величину

$$
d_{2}(v, w)=\max \left\{\left(\sum_{i=1}^{\operatorname{dim} V_{1}} w_{i}^{2}\right)^{\frac{1}{2}},\left(\sum_{i=\operatorname{dim} V_{1}+1}^{N} w_{i}^{2}\right)^{\frac{1}{4}}\right\} .
$$

Свойство 1. Величина $d_{2}(v, w)$ локально является квазиметрикой: она неотрицательна (и равна нулю тогда и только тогда, когда $v=w$ ), обладает свойством симметричности, и для всякой окрестности $D \subset \mathbb{G}$ существует константа $C_{D}<\infty$ такая, что обобщенное неравенство треугольника

$$
d_{2}(v, w) \leq C_{D}\left(d_{2}(v, u)+d_{2}(u, w)\right)
$$

верно для всех $v, u, w \in D$.

Шар в квазиметрике $d_{2}$ радиуса $r$ с центром в точке $v$, равный $\left\{w \in \mathbb{G}: d_{2}(v, w)<r\right\}$, обозначим символом $\operatorname{Box}_{2}(v, r)$.

Свойство 2. Рассмотрим точку $v \in \mathbb{G}$ и $\left(w_{1}, \ldots, w_{N}\right) \in \mathbb{R}^{N}$. Определим отображение $\theta_{v}: \mathbb{R}^{N} \rightarrow \mathbb{G}$ следующим образом:

$$
\theta_{v}\left(w_{1}, \ldots, w_{N}\right)=\exp \left(\sum_{i=1}^{N} w_{i} X_{i}\right)(v) .
$$

Известно, что $\theta_{v}$ - гладкий диффеоморфизм.

Определение 6. Набор $\left\{w_{i}\right\}_{i=1}^{N}$ называется нормалъными координатами (или координатами первого рода) (относителъно $v \in \mathbb{G})$ точки $w=\theta_{v}\left(w_{1}, \ldots, w_{N}\right)$.

Свойство 3. Образ шара $B_{0} x_{2}(v, r)$ при отображении $\theta_{v}^{-1}-$ декартово произведение шаров в евклидовой метрике, диаметры которых равны $2 r$ и $2 r^{2}$. 
Замечание 2. Образ шара в квазиметрике $d_{\infty}$ при отображении $\theta_{v}^{-1}$ равен декартову произведению кубов с длинами граней $2 r$ и $2 r^{2}$. При выводе свойств поверхностей, в частности, при подсчете их меры, возникает необходимость исследования пересечений соответствующих касательных плоскостей с субримановыми шарами. Так как форма пересечения шара с плоскостью всегда одна и та же (в отличие от случая кубов), то преимущество использования $d_{2}$ вместо $d_{\infty}$ очевидно.

Свойство 4. С помощью свойства 3 непосредственно проверяется, что хаусдорфова размерность группы $\mathbb{G}$ относительно $d_{2}$ равна $\nu=\operatorname{dim} V_{1}+2 \operatorname{dim} V_{2}$.

Перейдем к подробному описанию условий задачи и приведем необходимые понятия и результаты для отображений групп Карно.

Определение 7. Отображение $\varphi: \Omega \rightarrow \widetilde{\mathbb{G}}, \Omega \subset \mathbb{G}$, нильпотентных градуированных групп называется контактным отображением класса $C_{H}^{1}$, если его горизонтальные производные существуют и непрерывны, а отображение $\widehat{D}_{H} \varphi=\left(X_{1} \varphi, \ldots, X_{\operatorname{dim} V_{1}} \varphi\right)$ переводит горизонтальные поля в горизонтальные.

Свойство 5. Всякое контактное отображение класса $C_{H}^{1}$ является локально липшицевым во внутреннем смысле: если обозначить $d_{2}$-квазиметрику в образе символом $\widetilde{d}_{2}$, то локально верно соотношение $\widetilde{d}_{2}(\varphi(x), \varphi(y)) \leq L d_{2}(x, y), x, y \in \Omega$, для некоторого $L<\infty$, зависящего от рассматриваемой окрестности.

Определение 8 ([6], [7]). Пусть $\mathbb{G}-$ группа Карно, $\widetilde{\mathbb{G}}-$ нильпотентная градуированная группа, $D \subset \mathbb{G}-$ измеримое множество, и $\varphi: D \rightarrow \widetilde{\mathbb{G}}$. Отображение $\varphi$ является $h c$-дифференцируемым (или дифференцируемым в субримановом смысле) в (предельной) точке $x \in D$, если существует горизонтальный гомоморфизм $\mathcal{L}_{x}: \mathbb{G} \rightarrow \widetilde{\mathbb{G}}$ такой, что

$$
d_{2}\left(\varphi(y), \mathcal{L}_{x}\langle y\rangle\right)=o\left(d_{2}(x, y)\right) \text { при } y \rightarrow x, \quad y \in D .
$$

$h c$-дифференциал (или субриманов дифференциал) $\mathcal{L}_{x}$ обозначается символом $\widehat{D} \varphi(x)$.

Для липшицевых в субримановом смысле отображений справедлив аналог теоремы Радемахера о $h c$-дифференцируемости почти всюду липшицевых отображений ([6], см. также [7] для измеримых множеств). Для рассматриваемого класса отображений верно следующее утверждение, учитывающее непрерывную дифференцируемость по горизонтальным полям [7].

Теорема 1 ([7]). Пусть $\mathbb{G}$ - группа Карно, $\Omega \subset \mathbb{G}$ - открытое множество, $\widetilde{\mathbb{G}}-$ нильпотентная градуированная группа $и \varphi \in C_{H}^{1}(\Omega, \widetilde{\mathbb{G}})$. Тогда $\varphi$ непрерывно с-дифференцируемо всюду. Иными словами, элементы $(\widehat{D} \varphi)_{V_{1}, \widetilde{V}_{1}}(x),(\widehat{D} \varphi)_{V_{2}, \widetilde{V}_{2}}(x)$ субриманова дифференциала

$$
\left(\begin{array}{cc}
(\widehat{D} \varphi)_{V_{1}, \widetilde{V}_{1}}(x) & 0 \\
0 & (\widehat{D} \varphi)_{V_{2}, \widetilde{V}_{2}}(x)
\end{array}\right)
$$

непрерывно зависят от $x \in \Omega$.

Предположение 1. Будем рассматривать $\varphi: \Omega \rightarrow \widetilde{\mathbb{G}}$, где

(1) $\varphi: \Omega \rightarrow \widetilde{\mathbb{G}}$ - контактное отображение класса $C_{H}^{1} \cap C^{2}$;

(2) $\Omega \subset \mathbb{G}$ - открытое множество; 
(3) $\mathbb{G}, \widetilde{\mathbb{G}} \subset \mathbb{U}$, где $\mathbb{U}-$ двуступенчатая нильпотентная градуированная группа топологической размерности $N+\widetilde{n}+1$, с базисными полями $X_{1}, \ldots, X_{N}, \widetilde{X}_{1}, \ldots, \widetilde{X}_{\widetilde{n}+1}$, алгеброй Ли векторных полей $\widehat{V}=\widehat{V}_{1} \oplus \widehat{V}_{2}$, где поля $X_{1}, \ldots, X_{n}, \widetilde{X}_{1}, \ldots, \widetilde{X}_{\widetilde{n}}, n<N$, составляют базис $\widehat{V}_{1}$, поля $X_{n+1}, \ldots, X_{N}, \widetilde{X}_{\widetilde{n}+1}$ составляют базис $\widehat{V}_{2}$, и единицей $\widehat{\mathbf{0}}$;

(4) $\mathbb{G}$ - группа Карно топологической размерности $N$ с базисными полями $X_{1}, \ldots, X_{N}$, алгеброй Ли векторных полей $V=V_{1} \oplus V_{2}$, где поля $X_{1}, \ldots, X_{n}$ составляют базис $V_{1}$, поля $X_{n+1}, \ldots, X_{N}$ составляют базис $V_{2}$, и единицей $\mathbf{0}$;

(5) $\underset{\widetilde{\mathbb{G}}}{\widetilde{X}}-$ группа Карно топологической размерности $\widetilde{n}+1$ с базисными полями $\widetilde{X}_{1}, \ldots$, $\widetilde{X}_{\widetilde{n}+1}$, алгеброй Ли векторных полей $\widetilde{V}=\widetilde{V}_{1} \oplus \widetilde{V}_{2}$, где поля $\widetilde{X}_{1}, \ldots, \widetilde{X}_{\widetilde{n}}$ составляют базис $\widetilde{V}_{1}$, поле $\widetilde{X}_{\widetilde{n}+1}$ является базисным для $\widetilde{V}_{2}$, и единицей $\widetilde{\mathbf{0}}$;

(6) $\widehat{\mathbf{0}}=(\mathbf{0}, \widetilde{\mathbf{0}})$.

Замечание 3. Последнее условие предположения 1 означает, что, во-первых, пересечение $\mathbb{G}$ и $\widetilde{\mathbb{G}}$ совпадает с единицей группы $\mathbb{U}$, и, во-вторых, обе группы пересекаются по своим единицам.

Обозначение единиц групп нулями обусловлено тем, что многие рассуждения проводятся в нормальных координатах в том числе, относительно этих единиц (см. определение 6). При этом, очевидно, что их координаты относительно самих себя нулевые.

Определение 9. Пусть выполнены условия предположения 1. Отображение-график $\varphi_{\Gamma}: \Omega \rightarrow \mathbb{U}$ сопоставляет точке $x$ элемент

$$
\mathbb{U} \ni \varphi_{\Gamma}(x)=\exp \left(\sum_{j=1}^{\widetilde{N}} \varphi_{j}(x) \widetilde{X}_{j}\right)(x),
$$

где $\exp \left(\sum_{j=1}^{\widetilde{N}} \varphi_{j}(x) \widetilde{X}_{j}\right)(\widetilde{\mathbf{0}})=\varphi(x) \in \widetilde{\mathbb{G}}$

Для описания меры на поверхностях-графиках нам потребуются следующие определения и утверждения.

Определение 10 (например, [8]). Пусть $\mathbb{G}$ и $\widetilde{\mathbb{G}}-$ нильпотентные градуированные группы Ли, $\Omega \subset \mathbb{G}, \psi: \Omega \rightarrow \widetilde{\mathbb{G}}$, и функция $\widetilde{\mathfrak{d}}: \psi(\Omega) \times \widetilde{\mathbb{G}} \rightarrow \mathbb{R}_{+}$такова, что $\widetilde{\mathfrak{d}}(x, x)=0$ и $\widetilde{\mathfrak{d}}(x, y)=\widetilde{\mathfrak{d}}(y, x)$ для всех точек ее области определения. Будем говорить, что $\psi$ полиномиально субриманово дифферениируемо (или полиномиально hc-дифференцируемо) в (предельной) точке $x \in \Omega$ относительно $\widetilde{\mathfrak{d}}$, если существует отображение $\mathcal{L}_{x}: \mathbb{G} \rightarrow \widetilde{\mathbb{G}}$ такое, что

1) $\widetilde{\mathfrak{d}}\left(\psi(w), \mathcal{L}_{x}\langle w\rangle\right)=o\left(d_{2}(x, w)\right), \Omega \ni w \rightarrow x$

2) $\mathcal{L}_{x}(w)=\theta_{\psi(x)} \circ L_{x} \circ \theta_{x}^{-1}(w)$, где $L_{x}$ - полином по $w_{1}, \ldots, w_{N}$, a $\exp \left(\sum_{j=1}^{N} w_{j} X_{j}\right)(x)=w$.

Отображение $\mathcal{L}_{x}$ называется полиномиальным субримановым дифференииалом (или полиномиальным $h c$-дифференциалом) отображения $\psi$ в точке $x$.

Теорема 2 ([8]). График $\varphi_{\Gamma}$ контактного отображения $\varphi$ класса $C_{H}^{1}$ непрерывно полиномиально һс-дифферениируем всюду.

Из полученных в [8] для полиномиального $h c$-дифференциала $\widehat{D}_{P} \varphi_{\Gamma}$ выражений следует, что коэффициенты при полях второй степени будут сравнимы с $d_{2}$, а не с $\left(d_{2}\right)^{2}$. Кроме того, 
его дифференциал имеет вид

$$
D\left(\widehat{D}_{P} \varphi_{\Gamma}(x)\right)=\left(\begin{array}{cc}
E_{\operatorname{dim} V_{1}} & 0 \\
* & E_{\operatorname{dim} V_{2}} \\
(\widehat{D} \varphi)_{V_{1}, \widetilde{V}_{1}}(x) & 0 \\
* & (\widehat{D} \varphi)_{V_{2}, \widetilde{V}_{2}}(x)
\end{array}\right),
$$

где символ “*” обозначает элементы матрицы, которые могут не быть равными нулю, что существенно затрудняет вывод метрических свойств. Тем не менее, эта проблема решаема при помощи специального нового базиса $\left\{{ }^{x} X_{1}, \ldots,{ }^{x} X_{N},{ }^{x} \widetilde{X}_{1}, \ldots,{ }^{x} \widetilde{X}_{\widetilde{n}+1}\right\}$ в $\mathbb{U}$, который согласует неголономную структуру $\mathbb{U}$ с таковой у образов отображения-графика и его полиномиального субриманова дифференциала. Иными словами, образ $\widehat{D}_{P} \varphi_{\Gamma}(x)\left\langle B o x_{2}(x, r)\right\rangle$ полиномиального $h c$-дифференциала можно параметризовать следующим образом: он будет совпадать с множеством

$$
\left({ }^{x} \widetilde{\theta}_{\varphi_{\Gamma}(x)} \circ \theta_{\varphi_{\Gamma}(x)}^{-1}\right)\left(D_{\operatorname{diag}}\left(\widehat{D}_{P} \varphi_{\Gamma}(x)\right)(x)\left\langle B o x_{2}(x, r)\right\rangle\right),
$$

где ${ }^{x} \widetilde{\theta}_{\varphi_{\Gamma}(x)}$ - отображение координат первого рода в новом базисе, а $D_{\text {diag }}-$ блочно-диагональная часть матрицы дифференциала (в базисе, перенумерованном по степеням) с блоками размерностей $(n+\widetilde{n}) \times n$ и $(N-n+1) \times(N-n)$. Иными словами, множество $\widehat{D}_{P} \varphi_{\Gamma}(x)\left\langle B o x_{2}(x, r)\right\rangle$ параметризуется образом субриманова шара при отображении

$$
D_{\operatorname{diag}}\left(\widehat{D}_{P} \varphi_{\Gamma}(x)\right)(x)=\left(\begin{array}{cc}
E_{\operatorname{dim} V_{1}} & 0 \\
(\widehat{D} \varphi)_{V_{1}, \widetilde{V}_{1}}(x) & 0 \\
0 & E_{\operatorname{dim} V_{2}} \\
0 & (\widehat{D} \varphi)_{V_{2}, \widetilde{V}_{2}}(x)
\end{array}\right) .
$$

Определение 11. Если $w=\exp \left(\sum_{i=1}^{N} w_{i}{ }^{x} X_{i}+\sum_{j=1}^{\widetilde{n}+1} w_{N+j}{ }^{x} \widetilde{X}_{j}\right)(v)$, то

$$
{ }^{x} d_{2}(v, w)=\max \left\{\left(\sum_{i=1}^{n} w_{i}^{2}+\sum_{j=N+1}^{N+\widetilde{n}} w_{j}^{2}\right)^{\frac{1}{2}},\left(\sum_{i=n+1}^{N} w_{i}^{2}+w_{N+\widetilde{n}+1}^{2}\right)^{\frac{1}{4}}\right\} .
$$

Шар с центром в $v$ радиуса $r>0$ обозначим символом ${ }^{x} B_{0} x_{2}(v, r)$.

Опишем внутреннюю меру на поверхностях-графиках.

Определение 12. Пусть выполнены условия предположения 1. Значение внутренней меpы $\mathcal{H}_{\Gamma}^{\nu}$ для подмножеств $A \subset \varphi_{\Gamma}(\Omega)$ вычисляется по формуле

$$
\mathcal{H}_{\Gamma}^{\nu}(A)=\omega_{n} \omega_{N-n} \cdot \lim _{\delta \rightarrow 0} \inf \left\{\sum_{i \in \mathbb{N}} r_{i}^{\nu}: \bigcup_{i \in \mathbb{N}}^{\varphi_{\Gamma}^{-1}\left(x_{i}\right)} \operatorname{Box}_{2}\left(x_{i}, r_{i}\right) \supset A, x_{i} \in A, r_{i}<\delta\right\},
$$

где точная нижняя грань берется по всем покрытиям множества $A$.

Базовым результатом для исследований классов минимальных поверхностей является

Теорема 3 (см. также [9]). Пустъ выполнены условия предположения 1. Тогда для отображений-графиков справедлива формула площади

$$
\int_{\Omega} \mathcal{J}\left(\varphi_{\Gamma}, x\right) d \mathcal{H}^{\nu}(x)=\int_{\varphi_{\Gamma}(\Omega)} d \mathcal{H}_{\Gamma}^{\nu}(y)
$$


¿de

$$
\mathcal{J}\left(\varphi_{\Gamma}, x\right)=\sqrt{\operatorname{det}\left(E_{n}+(\widehat{D} \varphi)_{V_{1}, \widetilde{V}_{1}}^{*}(x)(\widehat{D} \varphi)_{V_{1}, \widetilde{V}_{1}}(x)\right)} \cdot \sqrt{1+(\widehat{D} \varphi)_{V_{2}, \widetilde{V}_{2}}^{*}(x)(\widehat{D} \varphi)_{V_{2}, \widetilde{V}_{2}}(x)} .
$$

\section{2. УСЛОВИЯ КОРРЕКТНОЙ ФОРМУЛИРОВКИ ЗАДАЧИ}

При исследовании поверхностей в пятимерном пространстве, в [3] установлено, что вариация отображения (как аргумента функционала площади) зависит только от горизонтальных координат отображения. Поэтому для каждого $\varphi: \Omega \rightarrow \widetilde{\mathbb{G}}$ будем строить вариации, определяемые исходным отображением и набором $\xi_{k}: \Omega \rightarrow \mathbb{R}, k=1, \ldots, \widetilde{n}$. В отличие от пятимерного случая, когда отображение было определено на группе Гейзенберга с единственным нетривиальным коммутатором базисных полей, на произвольных двуступенчатых группах Карно существует более одного не равного нулю коммутатора. Так как вариации аргумента функционала площади на неголономных структурах зависят от коммутаторов полей и их соотношений, то на выбор определяющих вариацию отображения функций $\xi_{k}$, $k=1, \ldots, \widetilde{n}$, могут возникнуть некоторые ограничения. Поэтому в данном разделе опишем условия корректности постановки задачи, т. е. условия отсутствия ограничений в выборе $\xi_{k}, k=1, \ldots, \widetilde{n}$.

Теорема 4. Для того чтобы не было ограничений в выборе $\xi_{k}, k=1, \ldots, \widetilde{n}$, необходимо и достаточно, чтобы все коммутаторы первого порлдка горизонтальных полей были либо линейно независимы с остальными, либо равны нулю.

Иными словами, на $\mathbb{G}$ вслкое поле степени два должно быть представимо единственным образом через коммутаторы горизонталъных полей.

Пример 1. Простейшим примером группы, удовлетворяющей условиям теоремы 4, является группа Гейзенберга $\mathbb{H}^{1}$ топологической размерности три с полями $X, Y, T$, где $[X, Y]=T$, определенными на $\mathbb{R}^{3}$. В качестве более сложного примера можно представить декартово произведение $k$ экземпляров $\mathbb{R}^{3}$, на каждом из которых определены поля $X_{j}, Y_{j}, T_{j}$, где $\left[X_{j}, Y_{j}\right]=T_{j}$. Тогда по построению имеем $\left[X_{j}, Y_{m}\right]=\left[X_{j}, X_{m}\right]=\left[Y_{j}, Y_{m}\right]=0$ для $m \neq j$, $j, m=1, \ldots, k$. Кроме того, можно рассмотреть декартово произведение $\mathbb{R}^{3} \times \mathbb{R}$, где на $\mathbb{R}^{3}$ определена структура группы Гейзенберга, а на $\mathbb{R}$ - постоянное векторное поле $Z$. В этом случае, полагая поля $X, Y, Z$ горизонтальными, получим группу, удовлетворяющую условиям теоремы 4. Действительно, по построению $[X, Z]=[Y, Z]=0$ и $[X, Y]=T$.

Доказательство теоремы 4. Как установлено в [2], [3], вариация определяющего график отображения определяется изменением его горизонтальных координат $\varphi_{1}, \ldots, \varphi_{\widetilde{n}}$, где

$$
\varphi(x)=\exp \left(\sum_{j=1}^{\widetilde{n}+1} \varphi_{j}(x) \widetilde{X}_{j}\right)(\widetilde{0})
$$

Пусть $k>n$. Тогда если $X_{k}=\sum_{i, j=1}^{n} a_{i, j}^{k}\left[X_{i}, X_{j}\right]$ (здесь и далее $i<j$ ), то для $x \in \Omega$ имеем

$$
\widehat{D} \varphi(x)\left\langle X_{k}\right\rangle=\sum_{i, j=1}^{n} a_{i, j}^{k} \widehat{D} \varphi(x)\left\langle\left[X_{i}, X_{j}\right]\right\rangle=\sum_{i, j=1}^{n} a_{i, j}^{k}\left[\widehat{D} \varphi(x)\left\langle X_{i}\right\rangle, \widehat{D} \varphi(x)\left\langle X_{j}\right\rangle\right] .
$$


Кроме того, при $\widehat{D} \varphi(x)\left\langle X_{i}\right\rangle=\sum_{l=1}^{\widetilde{n}}(\widehat{D} \varphi(x))_{l i} \widetilde{X}_{l}, i=1, \ldots, n$, имеем

$$
\begin{gathered}
\sum_{i, j=1}^{n} a_{i, j}^{k}\left[\widehat{D} \varphi(x)\left\langle X_{i}\right\rangle, \widehat{D} \varphi(x)\left\langle X_{j}\right\rangle\right]=\sum_{i, j=1}^{n} a_{i, j}^{k}\left[\sum_{l=1}^{\widetilde{n}}(\widehat{D} \varphi(x))_{l i} \widetilde{X}_{l}, \sum_{m=1}^{\widetilde{n}}(\widehat{D} \varphi(x))_{m j} \widetilde{X}_{m}\right]= \\
=\sum_{i, j=1}^{n} a_{i, j}^{k} \sum_{l, m=1}^{\widetilde{n}}\left((\widehat{D} \varphi(x))_{l i}(\widehat{D} \varphi(x))_{m j}-(\widehat{D} \varphi(x))_{m i}(\widehat{D} \varphi(x))_{l j}\right)\left[\widetilde{X}_{l}, \widetilde{X}_{m}\right]= \\
=\sum_{i, j=1}^{n} a_{i, j}^{k} \sum_{l, m=1}^{\widetilde{n}}\left((\widehat{D} \varphi(x))_{l i}(\widehat{D} \varphi(x))_{m j}-(\widehat{D} \varphi(x))_{m i}(\widehat{D} \varphi(x))_{l j}\right) c_{l m \widetilde{n}+1} \widetilde{X}_{\widetilde{n}+1},
\end{gathered}
$$

где $i<j$ и $l<m$. Таким образом, значение $\widehat{D} \varphi(x)\left\langle X_{k}\right\rangle$ определяется однозначно и выражается в виде (5). Покажем, что условие единственности представления поля второй степени через коммутаторы горизонтальных полей является необходимым. Заменим горизонтальные координатные функции $\varphi_{j}$ на $\psi_{\varepsilon, j}=\varphi_{j}+\varepsilon \xi_{j}$, где $\xi_{j}: \Omega \rightarrow \mathbb{R}, j=1, \ldots, \widetilde{n}$. Тогда

$$
\begin{aligned}
\widehat{D} \psi_{\varepsilon, j}(x)\left\langle X_{k}\right\rangle & =\sum_{i, j=1}^{n} a_{i, j}^{k} \sum_{l, m=1}^{\widetilde{n}}\left[\left((\widehat{D} \varphi(x))_{l i}+\varepsilon X_{i} \xi_{l}(x)\right)\left((\widehat{D} \varphi(x))_{m j}+\varepsilon X_{j} \xi_{m}(x)\right)-\right. \\
& \left.-\left((\widehat{D} \varphi(x))_{m i}+\varepsilon X_{i} \xi_{m}(x)\right)\left((\widehat{D} \varphi(x))_{l j}+\varepsilon X_{j} \xi_{l}(x)\right)\right] c_{l m \widetilde{n}+1} \widetilde{X}_{\widetilde{n}+1}= \\
= & \sum_{i, j=1}^{n} a_{i, j}^{k} \sum_{l, m=1}^{\widetilde{n}}\left((\widehat{D} \varphi(x))_{l i}(\widehat{D} \varphi(x))_{m j}-(\widehat{D} \varphi(x))_{m i}(\widehat{D} \varphi(x))_{l j}\right) c_{l m \tilde{n}+1} \widetilde{X}_{\widetilde{n}+1}+ \\
+ & \varepsilon \sum_{i, j=1}^{n} a_{i, j}^{k} \sum_{l, m=1}^{\widetilde{n}}\left((\widehat{D} \varphi(x))_{l i} X_{j} \xi_{m}(x)-(\widehat{D} \varphi(x))_{l j} X_{i} \xi_{m}(x)\right) c_{l m \widetilde{n}+1} \widetilde{X}_{\widetilde{n}+1}+ \\
+ & \varepsilon \sum_{i, j=1}^{n} a_{i, j}^{k} \sum_{l, m=1}^{\widetilde{n}}\left((\widehat{D} \varphi(x))_{m j} X_{i} \xi_{l}(x)-(\widehat{D} \varphi(x))_{m i} X_{j} \xi_{l}(x)\right) c_{l m \widetilde{n}+1} \widetilde{X}_{\widetilde{n}+1}+ \\
& +\varepsilon^{2} \sum_{i, j=1}^{n} a_{i, j}^{k} \sum_{l, m=1}^{\tilde{n}}\left(X_{i} \xi_{l}(x) X_{j} \xi_{m}(x)-X_{i} \xi_{m}(x) X_{j} \xi_{l}(x)\right) c_{l m \widetilde{n}+1} \widetilde{X}_{\widetilde{n}+1} .
\end{aligned}
$$

Отметим, здесь $i<j$ и $l<m$. Фиксируем точку $x \in \Omega$ и перейдем к нормальным координатам относительно нее. Далее фикисируем $k$, а также такие $m$ и $l$, что $c_{l m \tilde{n}+1} \neq 0$, и рассмотрим (в координатах первого рода) функции $\xi_{l}=x_{p}$ и $\xi_{m}=x_{q}$, а остальные положим равными нулю. Тогда в точке $0 \in \mathbb{R}^{N}$ имеем $\left(\theta_{u}^{-1}\right)_{*} X_{p} \xi_{l}(0)=\left(\theta_{u}^{-1}\right)_{*} X_{q} \xi_{m}(0)=1$, а остальные соотношения равны нулю. В частности, последнее слагаемое в (6) равно

$$
\varepsilon^{2} a_{p, q}^{k} c_{l m \tilde{n}+1} \widetilde{X}_{\widetilde{n}+1} \neq 0 .
$$

Поэтому при предположении

выводим

$$
X_{k}=\sum_{i, j=1}^{n} a_{i, j}^{k}\left[X_{i}, X_{j}\right]=\sum_{i, j=1}^{n} \bar{a}_{i, j}^{k}\left[X_{i}, X_{j}\right]
$$

$$
\varepsilon \cdot a_{p, q}^{k} S+\varepsilon^{2} a_{p, q}^{k} c_{l m \widetilde{n}+1}=\varepsilon \cdot \bar{a}_{p, q}^{k} S+\varepsilon^{2} \bar{a}_{p, q}^{k} c_{l m \widetilde{n}+1}
$$


где величина $S$ зависит только от точки $x$. Так как $\varepsilon>0$ произвольно, то получаем $a_{p, q}^{k}=\bar{a}_{p, q}^{k}$ для всех возможных $k, p, q$. Иными словами, всякое поле степени два в прообразе должно быть представимо единственным образом через коммутаторы горизонтальных полей. Достаточность такого условия очевидна из (6).

В силу установленных результатов, к предположению 1 добавляется

Предположение 2. На $\mathbb{G}$ всякое поле степени два представимо в виде линейной комбинации коммутаторов горизонтальных полей единственным образом.

\section{3. МИНИМАЛЬНЫЕ ПОВЕРХНОСТИ И СВОЙСТВА}

Положим для субриманова дифференциала $\widehat{D}_{H} \varphi(x)=(\widehat{D} \varphi)_{V_{1}, \widetilde{V}_{1}}(x)$ и $\widehat{D}_{H^{\perp}} \varphi(x)=$ $=(\widehat{D} \varphi)_{V_{2}, \widetilde{V}_{2}}(x)$. Таким образом, матрица субриманова дифференциала в точке $x \in \Omega$ имеет вид

$$
\left(\begin{array}{cc}
\widehat{D}_{H} \varphi(x) & 0 \\
0 & \widehat{D}_{H^{\perp}} \varphi(x)
\end{array}\right) .
$$

Определение 13. Функционалом площади, действующим на классе графиков, построенных по контактным отображениям класса $C_{H}^{1} \cap C^{2}$, называется выражение

$$
S(\varphi)=\int_{\Omega} \sqrt{\operatorname{det}\left(E_{n}+\widehat{D}_{H} \varphi(x)^{*} \widehat{D}_{H} \varphi(x)\right)} \cdot \sqrt{1+\left\langle\widehat{D}_{H^{\perp}} \varphi(x), \widehat{D}_{H^{\perp}} \varphi(x)\right\rangle} d \mathcal{H}^{\nu}(x) .
$$

Здесь и далее верхний индекс “*” обозначает сопряженный оператор, а $\langle\cdot, \cdot\rangle-$ скалярное произведение.

Замечание 4. Функционал площади (7) определен корректно и для произвольных липшицевых в субримановом смысле отображений.

Обозначим символом $\widehat{D} \psi_{\varepsilon}(x)$ горизонтальный гомоморфизм, зависящий от точки $x \in \Omega$, значения которого определяются горизонтальными производными функций $\psi_{\varepsilon, j}=\varphi_{j}+\varepsilon \xi_{j}$ и соотношением (6) доказательства теоремы 4 . Из него получаем условие на приращение функционала площади. А именно, для полей $X_{k}$ второй степени имеем

$$
\begin{aligned}
\widehat{D} \psi_{\varepsilon}(x)\left\langle X_{k}\right\rangle & =\widehat{D} \varphi(x)\left\langle X_{k}\right\rangle+ \\
+ & \varepsilon \sum_{i, j=1}^{n} a_{i, j}^{k} \sum_{l, m=1}^{\widetilde{n}}\left((\widehat{D} \varphi(x))_{l i} X_{j} \xi_{m}(x)-(\widehat{D} \varphi(x))_{l j} X_{i} \xi_{m}(x)\right) c_{l m \widetilde{n}+1} \widetilde{X}_{\widetilde{n}+1}+ \\
+ & \varepsilon \sum_{i, j=1}^{n} a_{i, j}^{k} \sum_{l, m=1}^{\widetilde{n}}\left((\widehat{D} \varphi(x))_{m j} X_{i} \xi_{l}(x)-(\widehat{D} \varphi(x))_{m i} X_{j} \xi_{l}(x)\right) c_{l m \widetilde{n}+1} \widetilde{X}_{\widetilde{n}+1}+ \\
& +\varepsilon^{2} \sum_{i, j=1}^{n} a_{i, j}^{k} \sum_{l, m=1}^{\widetilde{n}}\left(X_{i} \xi_{l}(x) X_{j} \xi_{m}(x)-X_{i} \xi_{m}(x) X_{j} \xi_{l}(x)\right) c_{l m \widetilde{n}+1} \widetilde{X}_{\widetilde{n}+1}= \\
& =\phi_{k}(x) \widetilde{X}_{\widetilde{n}+1}+\left(\varepsilon P_{1}^{k}\left(\widehat{D}_{H} \varphi(x), \widehat{D}_{H} \xi(x)\right)+\varepsilon^{2} P_{2}^{k}\left(\widehat{D}_{H} \xi(x)\right)\right) \widetilde{X}_{\widetilde{n}+1},
\end{aligned}
$$

где $i<j$ и $l<m, \phi_{k}-$ коэффициент при $\widetilde{X}_{\widetilde{n}+1}$ в $\widehat{D} \varphi(x)\left\langle X_{k}\right\rangle, P_{1}^{k}-$ полином от горизонтальных производных функций $\xi_{1}, \ldots, \xi_{\widetilde{n}}$ степени один, а $P_{k}^{2}-$ полином от горизонтальных производных функций $\xi_{1}, \ldots, \xi_{\widetilde{n}}$ степени два, $k=n+1, \ldots, N$. Здесь и далее символ $\widehat{D}_{H} \xi$ 
обозначает матрицу из горизонтальных производных функций $\xi_{q}$, элемент которой с номером $(q, p)$ равен $X_{p} \xi_{q}, q=1, \ldots, \widetilde{n}, p=1, \ldots, n$.

Определение 14. Приращение функционала площади на элементе $\xi=\left(\xi_{1}, \ldots, \xi_{\widetilde{n}}\right)$ будет равно $S\left(\widehat{D} \psi_{\varepsilon}\right)-S(\varphi)$, где $S\left(\widehat{D} \psi_{\varepsilon}\right)=\int_{\Omega} \sqrt{\operatorname{det}\left(E_{n}+\left(\widehat{D}_{H} \varphi(x)+\varepsilon D_{H} \xi(x)\right)^{*}\left(\widehat{D}_{H} \varphi(x)+\varepsilon D_{H} \xi(x)\right)\right)} \times$ $\times \sqrt{1+\Delta^{2}(x)} d \mathcal{H}^{\nu}(x)$, и

$$
\Delta^{2}(x)=\sum_{k=n+1}^{N}\left(\phi_{k}(x)+\varepsilon P_{1}^{k}\left(\widehat{D}_{H} \varphi(x), \widehat{D}_{H} \xi(x)\right)+\varepsilon^{2} P_{2}^{k}\left(\widehat{D}_{H} \xi(x)\right)\right)^{2} .
$$

Замечание 5. Фактически, аргументом функционала площади являются не отображения, а их субримановы дифференциалы или, в более общем случае, горизонтальные гомоморфизмы, зависящие от точек прообраза. Подчеркнем, что отображения $\psi_{\varepsilon}$, субриманов дифференциал которого равен $\widehat{D} \psi_{\varepsilon}(x)$ в почти каждой точке, может не существовать. Следовательно, понятия "минимальная площадь" и "минимум функционала площади" различны.

Введем теперь норму для отображений.

Определение 15. Пусть $\Omega \subset \mathbb{G}, \xi_{1}, \ldots, \xi_{\widetilde{n}} \in C_{H}^{1}(\Omega, \mathbb{R})$, и $m \in \mathbb{N}$. Для $\xi=\left(\xi_{1}, \ldots, \xi_{\tilde{n}}\right)$ равенством определим норму

$$
\|\xi\|_{m}=\left(\int_{\Omega} \sum_{k=1}^{\widetilde{n}}\left|\xi_{k}(x)\right|^{m}+\sum_{\beta:|\beta|=m}\left|\widehat{\xi}(x)^{\beta}\right| d \mathcal{H}^{\nu}(x)\right)^{\frac{1}{m}},
$$

а для $\xi=\left(\xi_{1}, \ldots, \xi_{\widetilde{n}}\right)-($ полу $)$ норму

$$
\|\xi\|_{H, m}=\left(\int_{\Omega} \sum_{\beta:|\beta|=m}\left|\widehat{\xi}(x)^{\beta}\right| d \mathcal{H}^{\nu}(x)\right)^{\frac{1}{m}}
$$

где $\widehat{\xi}$ - вектор-функция, состоящая из элементов

$$
X_{1} \xi_{1}, \ldots, X_{1} \xi_{\widetilde{n}}, X_{2} \xi_{1}, \ldots, X_{n} \xi_{\widetilde{n}}
$$

Пусть $B$ - матрица. Символ $\|B\|_{H, m}$ обозначает выражение (8) с заменой подинтегрального выражения на сумму модулей всевозможных произведений элементов $B$ из $m$ множителей.

Теорема 5. Функционал площади (7) является дифференцируемым относительно нормы $\|\cdot\|_{4 n}$.

Доказательство. Проведем сначала рассуждения для $n=2$. Положим $E=E_{n}$ и исследуем на дифференцируемость функцию

$$
f(\varepsilon)=\sqrt{\operatorname{det}\left(E+(A+\varepsilon B)^{*}(A+\varepsilon B)\right)} \cdot \sqrt{1+\sum_{k=n+1}^{N}\left(c_{k}+\varepsilon P_{1}^{k}\left(a_{i j}, b_{m l}\right)+\varepsilon^{2} P_{2}^{k}\left(b_{m l}\right)\right)^{2}},
$$

$i, j=1, \ldots, n, i<j, l, m=1, \ldots, \widetilde{n}, l<m$. Так как $\left|f(\varepsilon)-f(0)-f^{\prime}(0) \varepsilon\right| \leq \frac{\varepsilon^{2}}{2}\left|f^{\prime \prime}(\widetilde{\varepsilon})\right|$, $\widetilde{\varepsilon} \in(0, \varepsilon)$, то для доказательства дифференцируемости найдем вторую производную. Для удобства выпишем сначала производные каждого множителя. Обозначим

$$
f_{1}(\varepsilon)=\sqrt{\operatorname{det}\left(E+(A+\varepsilon B)^{*}(A+\varepsilon B)\right)}=\sqrt{f_{2}(\varepsilon)},
$$




$$
f_{3}(\varepsilon)=\sqrt{1+\sum_{k=n+1}^{N}\left(c_{k}+\varepsilon P_{1}^{k}\left(a_{i j}, b_{m l}\right)+\varepsilon^{2} P_{2}^{k}\left(b_{m l}\right)\right)^{2}}=\sqrt{f_{4}(\varepsilon)} .
$$

Кроме того, представим матрицы $A$ и $B$ в виде наборов столбцов:

$$
A=\left(a_{1}, \ldots, a_{n}\right), \quad B=\left(b_{1}, \ldots, b_{n}\right) .
$$

Тогда $f_{2}^{\prime}(\varepsilon)$ - сумма $n$ слагаемых, где каждое слагаемое с номером $i$ представляет собой определитель матрицы $E+(A+\varepsilon B)^{*}(A+\varepsilon B)$; здесь строку с номером $i$ заменили строкой с номером $i$ этой же матрицы, элементы которой продифференцированы по $\varepsilon, i=1, \ldots, n$. Эта строка равна $b_{i}^{*} A+a_{i}^{*} B+2 \varepsilon b_{i}^{*} B, i=1, \ldots, n$. Обозначим матрицу с такой замененной строкой символом $A_{B}^{\prime}(i, \varepsilon), i=1, \ldots, n$. Тогда при $\varepsilon=0$ с помощью разложения определителя по строке и с учетом линейности получаем

$$
\left.f_{2}^{\prime}(\varepsilon)\right|_{\varepsilon=0}=\sum_{i=1}^{n} \sum_{j=1}^{n}\left\langle b_{i}, a_{j}\right\rangle\left(E+A^{*} A\right)_{i j}+\sum_{i=1}^{n} \sum_{j=1}^{n}\left\langle a_{i}, b_{j}\right\rangle\left(E+A^{*} A\right)_{i j},
$$

так как элементы строки с номером $i$ равны $\left\langle b_{i}, a_{j}\right\rangle+\left\langle a_{i}, b_{j}\right\rangle$, где $\left(E+A^{*} A\right)_{i j}$ - алгебраические дополнения, $i, j=1, \ldots, n$.

В случае рассмотрения второй производной определителя, получим $n^{2}$ слагаемых, каждое из которых равно определителю матрицы $A_{B}^{\prime}(i, \varepsilon)$, в которой одну из строк заменили строкой, состоящей из производных ее элементов. Иными словами, это матрица $E+(A+$ $\varepsilon B)^{*}(A+\varepsilon B)$, в которой либо строки $i$ и $j, i \neq j$, равны соответственно $b_{i}^{*} A+a_{i}^{*} B+2 \varepsilon b_{i}^{*} B$ и $b_{j}^{*} A+a_{j}^{*} B+2 \varepsilon b_{j}^{*} B$, либо строка с номером $i$ равна $2 b_{i}^{*} B, i, j=1, \ldots, n$. Матрицы, полученные в результате замены двух строк, обозначим символом $A_{B}^{\prime \prime}(i, j, \varepsilon), i, j=1, \ldots, n, i \neq j$, а одной - символом $A_{B}^{\prime \prime}(i, i, \varepsilon), i=1, \ldots, n$. Заметим, что вторая производная определителя оценивается как

$$
O\left(\sum_{i, j} b_{i j}^{2}\right)
$$

если $\varepsilon=0$. В случае, когда $\varepsilon \neq 0$, то появляются еще слагаемые

$$
O\left(\varepsilon \sum b_{i_{1} j_{1}} b_{i_{2} j_{2}} b_{i_{3} j_{3}}\right)+O\left(\varepsilon^{2} \sum b_{i_{1} j_{1}} b_{i_{2} j_{2}} b_{i_{3} j_{3}} b_{i_{4} j_{4}}\right),
$$

но так как элементы $B$ ограничены, а $\varepsilon>0$ мало, то и они оцениваются через (10). Тем не менее, в силу присутствия слагаемых (11) дифференцируемость будем устанавливать по норме, как минимум, $\|\cdot\|_{4}$.

Далее, очевидно,

$$
\left.f_{4}^{\prime}\right|_{\varepsilon=0}=2 \sum_{k=n+1}^{N} c_{k} P_{1}^{k}\left(a_{i j}, b_{m l}\right)
$$

Кроме того,

$$
f_{4}^{\prime \prime}(\varepsilon)=2 \sum_{k=n+1}^{N}\left(\left(P_{1}^{k}\left(a_{i j}, b_{m l}\right)\right)^{2}+2 c_{k} P_{2}^{k}\left(b_{m l}\right)+6 \varepsilon P_{1}^{k}\left(a_{i j}, b_{m l}\right) P_{2}^{k}\left(b_{m l}\right)+6 \varepsilon^{2}\left(P_{2}^{k}\left(b_{m l}\right)\right)^{2}\right) .
$$

Перейдем непосредственно к исследованию дифференцируемости функционала. Найдем оценку сверху $\left(f_{1}(\varepsilon) f_{3}(\varepsilon)\right)^{\prime \prime}=\left(\sqrt{f_{2}(\varepsilon)} \sqrt{f_{4}(\varepsilon)}\right)^{\prime \prime}$. Она выражается в виде

$$
\frac{2 f_{2}^{\prime \prime} f_{2}-\left(f_{2}^{\prime}\right)^{2}}{2 f_{2}^{3 / 2}}+2 \frac{f_{2}^{\prime}}{2 \sqrt{f_{2}}} \frac{f_{4}^{\prime}}{2 \sqrt{f_{4}}}+\frac{2 f_{4}^{\prime \prime} f_{4}-\left(f_{4}^{\prime}\right)^{2}}{2 f_{4}^{3 / 2}} \text {. }
$$


Так как $(A+\varepsilon B)^{*}(A+\varepsilon B)=Q D_{\varepsilon} Q^{T}, Q$ - ортогональная матрица, а $D_{\varepsilon}$ - диагональная матрица с неотрицательными элементами (в силу того, что матрица $(A+\varepsilon B)^{*}(A+\varepsilon B)$ положительно определена), то

$$
E+(A+\varepsilon B)^{*}(A+\varepsilon B)=Q\left(E+D_{\varepsilon}\right) Q^{T},
$$

и поэтому $f_{2} \geq 1$. Кроме того, по определению $f_{4} \geq 1$. Из оценок (9)-(13) следует

$$
\begin{aligned}
\left|\left(f_{1}(\varepsilon) f_{3}(\varepsilon)\right)^{\prime \prime}\right| \leq K_{1}\left(\sum_{i, j} b_{i j}^{2}\right)+\varepsilon K_{2}\left(\sum\left|b_{i_{1} j_{1}} b_{i_{2} j_{2}} b_{i_{3} j_{3}}\right|\right)+ & \varepsilon^{2} K_{3}\left(\sum\left|b_{i_{1} j_{1}} b_{i_{2} j_{2}} b_{i_{3} j_{3}} b_{i_{4} j_{4}}\right|\right)+ \\
& +O\left(\varepsilon^{2}\right)\left(\sum_{l=5}^{8} K_{l} \prod_{k=1}^{l}\left|b_{i_{k} j_{k}}\right|\right)
\end{aligned}
$$

Тогда для $\Omega \Subset \mathbb{G}$ имеем дифференцирумость функционала площади относительно нормы $\|\cdot\|_{8}$ (для первых двух слагаемых соотношения (14) достаточно применить неравенство Гёльдера для интегралов):

$$
\begin{gathered}
\left|\int_{\Omega}\right| b_{i_{1} j_{1}} b_{i_{2} j_{2}} b_{i_{3} j_{3}}|d x| \leq\left|\int_{\Omega}\left(b_{i_{1} j_{1}} b_{i_{2} j_{2}}\right)^{2} d x\right|^{1 / 2}\left|\int_{\Omega}\left(b_{i_{3} j_{3}}\right)^{2} d x\right|^{1 / 2} \leq \\
\leq K(\Omega)\left|\int_{\Omega}\left(b_{i_{1} j_{1}} b_{i_{2} j_{2}}\right)^{4} d x\right|^{1 / 4}\left|\int_{\Omega}\left(b_{i_{3} j_{3}}\right)^{8} d x\right|^{1 / 8} \leq K(\Omega)\|B\|_{H, 8}^{3} .
\end{gathered}
$$

Окончательно, в силу оценки (10) получаем

$$
\left|f(\varepsilon)-f(0)-f^{\prime}(0) \varepsilon\right| \leq C(\Omega)\|B\|_{H, 8} .
$$

Рассуждения для произвольного $n>2$ проводятся аналогично с учетом того, что в соотношениях вида (11) и (14) полином от элементов матрицы $B$ будет иметь степень $2 n$ вместо 4 в силу правил вычисления определителя. Таким образом, функционал площади (7) дифференцируем относительно (полу)нормы $\|\cdot\|_{H, 4 n}$ и нормы $\|\cdot\|_{4 n}$.

Из установленного утверждения получаем следующий результат, являющийся одним из основных в статье.

Теорема 6. Для того чтобы функиионал площади достигал минимума на поверхностиграфике, определяемой контактным отображением $\varphi$ и горизонталъным гомоморфизмом $\widehat{D} \varphi$, среди значений, определяемых горизонтальными гомоморфизмами вида $\widehat{D} \psi_{\varepsilon}$ из обозначения 4, необходимо, чтобы был равен нулю интеграл

$$
\int_{\Omega} \mathcal{D}_{H}(\varphi, \xi) \frac{\sqrt{1+\left\langle\widehat{D} \varphi_{H^{\perp}}, \widehat{D} \varphi_{H^{\perp}}\right\rangle}}{\sqrt{\operatorname{det}\left(E_{n}+\widehat{D}_{H} \varphi^{*} \widehat{D}_{H} \varphi\right)}}+\mathcal{D}_{H^{\perp}}(\varphi, \xi) \frac{\sqrt{\operatorname{det}\left(E_{n}+\widehat{D}_{H} \varphi^{*} \widehat{D}_{H} \varphi\right)}}{\sqrt{1+\left\langle\widehat{D}_{H^{\perp}} \varphi, \widehat{D}_{H^{\perp}} \varphi\right\rangle}} d \mathcal{H}^{\nu},
$$

¿de

$$
\begin{aligned}
\mathcal{D}_{H}(\varphi, \xi, x)=\sum_{i=1}^{n} \sum_{j=1}^{n}\left\langle(D \xi)_{i},\left(\widehat{D}_{H} \varphi(x)\right)_{j}\right\rangle\left(E+\widehat{D}_{H} \varphi(x)^{*} \widehat{D}_{H} \varphi(x)\right)_{i j}+ \\
+\sum_{i=1}^{n} \sum_{j=1}^{n}\left\langle\left(\widehat{D}_{H} \varphi(x)\right)_{i},(D \xi)_{j}\right\rangle\left(E+\widehat{D}_{H} \varphi(x)^{*} \widehat{D}_{H} \varphi(x)\right)_{i j},
\end{aligned}
$$


кроме того,

$$
\begin{aligned}
& \mathcal{D}_{H^{\perp}}(\varphi, \xi, x)=2 \sum_{k=n+1}^{N}(\widehat{D} \varphi(x))_{\tilde{n}+1 k} \times \\
& \times\left(\sum_{i, j=1}^{n} a_{i, j}^{k} \sum_{l, m=1}^{\widetilde{n}}\left((\widehat{D} \varphi(x))_{l i} X_{j} \xi_{m}(x)-(\widehat{D} \varphi(x))_{l j} X_{i} \xi_{m}(x)\right) c_{l m \tilde{n}+1}+\right. \\
& \left.+\sum_{i, j=1}^{n} a_{i, j}^{k} \sum_{l, m=1}^{\tilde{n}}\left((\widehat{D} \varphi(x))_{m j} X_{i} \xi_{l}(x)-(\widehat{D} \varphi(x))_{m i} X_{j} \xi_{l}(x)\right) c_{l m \tilde{n}+1}\right),
\end{aligned}
$$

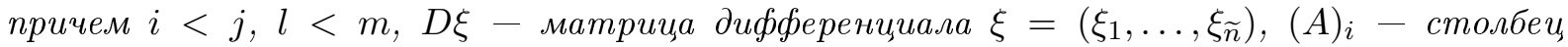
матрииь $A$ с номером $i$, а $A_{i j}$ - алгебраическое дополнение.

Замечание 6. Так как вариации для отображений с неголономным образом определены в общем случае не всегда, то понятия "максимальная площадь поверхности" и "максимум функционала площади" различны (см. подробности в [3], замечание 34).

Положим

$$
\mathcal{F}(\varphi)=\frac{\sqrt{1+\left\langle\widehat{D} \varphi_{H^{\perp}}, \widehat{D} \varphi_{H^{\perp}}\right\rangle}}{\sqrt{\operatorname{det}\left(E_{n}+\widehat{D}_{H} \varphi^{*} \widehat{D}_{H} \varphi\right)}}
$$

и преобразуем значения $\mathcal{D}_{H}(\varphi, \xi, x)$ и $\mathcal{D}_{H \perp}(\varphi, \xi, x)$, чтобы сформулировать определения субримановой средней кривизны и соответствующий критерий. Имеем

$$
\begin{aligned}
& \mathcal{D}_{H}(\varphi, \xi, x)=\sum_{i=1}^{n} \sum_{j=1}^{n} \sum_{k=1}^{\widetilde{n}}\left(X_{i} \xi_{k}(x) \cdot X_{j} \varphi_{k}(x)+X_{i} \varphi_{k}(x) \cdot X_{j} \xi_{k}(x)\right)\left(E+\widehat{D}_{H} \varphi(x)^{*} \widehat{D}_{H} \varphi(x)\right)_{i j}= \\
& =\sum_{i=1}^{n} \sum_{j=1}^{n} \sum_{k=1}^{\tilde{n}}\left(X_{i} \xi_{k}(x) \cdot X_{j} \varphi_{k}(x)\right)\left(E+\widehat{D}_{H} \varphi(x)^{*} \widehat{D}_{H} \varphi(x)\right)_{i j}+ \\
& +\sum_{i=1}^{n} \sum_{j=1}^{n} \sum_{k=1}^{\tilde{n}}\left(X_{i} \xi_{k}(x) \cdot X_{j} \varphi_{k}(x)\right)\left(E+\widehat{D}_{H} \varphi(x)^{*} \widehat{D}_{H} \varphi(x)\right)_{j i} \\
& \mathcal{D}_{H^{\perp}}(\varphi, \xi, x)=2 \sum_{k=n+1}^{N}(\widehat{D} \varphi(x))_{\tilde{n}+1, k} \sum_{i, j=1}^{n} a_{i, j}^{k} \sum_{l, m=1}^{\widetilde{n}} c_{l m \tilde{n}+1}\left(X_{i} \varphi_{l}(x) \cdot X_{j} \xi_{m}(x)-X_{j} \varphi_{l}(x) \cdot X_{i} \xi_{m}(x)\right)+ \\
& +2 \sum_{k=n+1}^{N}(\widehat{D} \varphi(x))_{\widetilde{n}+1, k} \sum_{i, j=1}^{n} a_{i, j}^{k} \sum_{l, m=1}^{\widetilde{n}} c_{l m \tilde{n}+1}\left(X_{j} \varphi_{m} \cdot X_{i} \xi_{l}(x)-X_{i} \varphi_{m}(x) \cdot X_{j} \xi_{l}(x)\right),
\end{aligned}
$$

где $i<j$ и $l<m$. Далее будем предполагать, что $\Omega \subset \mathbb{G}$ - область с достаточно гладкой границей, и $\left.\xi\right|_{\partial \Omega} \equiv 0$. Тогда при действии сопряженным к $X_{i}$ и $X_{j}$ оператором, который совпадает с $X_{i}$ и $X_{j}$ соответственно, и интегрировании по частям получаем, что в правой части суммы (15) каждое слагаемое меняется на

$$
X_{i}\left\langle X_{j} \varphi_{k}(x)\left(E+\widehat{D}_{H} \varphi(x)^{*} \widehat{D}_{H} \varphi(x)\right)_{i j} \mathcal{F}(\varphi)\right\rangle \cdot \xi_{k}
$$

или

$$
X_{i}\left\langle X_{j} \varphi_{k}(x)\left(E+\widehat{D}_{H} \varphi(x)^{*} \widehat{D}_{H} \varphi(x)\right)_{j i} \mathcal{F}(\varphi)\right\rangle \cdot \xi_{k},
$$


а в (16)

$$
\begin{aligned}
& 2 \sum_{k=n+1}^{N} \sum_{i, j=1}^{n} a_{i, j}^{k} \sum_{l, m=1}^{\widetilde{n}} c_{l m \widetilde{n}+1} X_{j}\left\langle X_{i} \varphi_{l}(x)(\widehat{D} \varphi(x))_{\widetilde{n}+1, k} \mathcal{F}(\varphi)^{-1}\right\rangle \xi_{m}(x)- \\
& \quad-2 \sum_{k=n+1}^{N} \sum_{i, j=1}^{n} a_{i, j}^{k} \sum_{l, m=1}^{\widetilde{n}} c_{l m \widetilde{n}+1} X_{i}\left\langle X_{j} \varphi_{l}(x)(\widehat{D} \varphi(x))_{\tilde{n}+1, k} \mathcal{F}(\varphi)^{-1}\right\rangle \xi_{m}(x)+ \\
& \quad+2 \sum_{k=n+1}^{N} \sum_{i, j=1}^{n} a_{i, j}^{k} \sum_{l, m=1}^{\tilde{n}} c_{l m \widetilde{n}+1} X_{i}\left\langle X_{j} \varphi_{m}(x)(\widehat{D} \varphi(x))_{\widetilde{n}+1, k} \mathcal{F}(\varphi)^{-1}\right\rangle \xi_{l}(x)- \\
& \quad-2 \sum_{k=n+1}^{N} \sum_{i, j=1}^{n} a_{i, j}^{k} \sum_{l, m=1}^{\widetilde{n}} c_{l m \widetilde{n}+1} X_{j}\left\langle X_{i} \varphi_{m}(x)(\widehat{D} \varphi(x))_{\widetilde{n}+1, k} \mathcal{F}(\varphi)^{-1}\right\rangle \xi_{l}(x),
\end{aligned}
$$

где $i<j$ и $l<m$.

Из приведенных выше рассуждений выводим понятие горизонтальной средней кривизны, которое является субримановым аналогом средней кривизны.

Определение 16. Вектором горизонталъной средней кривизны называется набор $H^{S R}(\varphi, x)=\left(H_{1}^{S R}(x), \ldots, H_{\widetilde{n}}^{S R}(x)\right)$, где

$$
\begin{aligned}
H_{m}^{S R}(x)=\sum_{i=1}^{n} \sum_{j=1}^{n} X_{i}\left\langle X_{j} \varphi_{m}(x)\left(E+\widehat{D}_{H} \varphi(x)^{*} \widehat{D}_{H} \varphi(x)\right)_{i j} \mathcal{F}(\varphi)\right\rangle+ \\
\quad+\sum_{i=1}^{n} \sum_{j=1}^{n} X_{i}\left\langle X_{j} \varphi_{m}(x)\left(E+\widehat{D}_{H} \varphi(x)^{*} \widehat{D}_{H} \varphi(x)\right)_{j i} \mathcal{F}(\varphi)\right\rangle+ \\
+2 \sum_{k=n+1}^{N} \sum_{i, j=1}^{n} a_{i, j}^{k} \sum_{1 \leq l<m} c_{l m \widetilde{n}+1} X_{j}\left\langle X_{i} \varphi_{l}(x)(\widehat{D} \varphi(x))_{\widetilde{n}+1, k} \mathcal{F}(\varphi)^{-1}\right\rangle- \\
\quad-2 \sum_{k=n+1}^{N} \sum_{i, j=1}^{n} a_{i, j}^{k} \sum_{1 \leq l<m} c_{l m \widetilde{n}+1} X_{i}\left\langle X_{j} \varphi_{l}(x)(\widehat{D} \varphi(x))_{\widetilde{n}+1, k} \mathcal{F}(\varphi)^{-1}\right\rangle+ \\
+2 \sum_{k=n+1}^{N} \sum_{i, j=1}^{n} a_{i, j}^{k} \sum_{m<l \leq \widetilde{n}} c_{m l \widetilde{n}+1} X_{i}\left\langle X_{j} \varphi_{l}(x)(\widehat{D} \varphi(x))_{\widetilde{n}+1, k} \mathcal{F}(\varphi)^{-1}\right\rangle- \\
\quad-2 \sum_{k=n+1}^{N} \sum_{i, j=1}^{n} a_{i, j}^{k} \sum_{m<l \leq \widetilde{n}} c_{m l \widetilde{n}+1} X_{j}\left\langle X_{i} \varphi_{l}(x)(\widehat{D} \varphi(x))_{\widetilde{n}+1, k} \mathcal{F}(\varphi)^{-1}\right\rangle
\end{aligned}
$$

$m=1, \ldots, \widetilde{n}$.

Из теоремы 6 и формулы интегрирования по частям получаем следующее необходимое условие минимальности.

Теорема 7. Для того чтобы функционал площади достигал минимума на поверхностиграфике, определяемой контактным $C^{2}$-отображением $\varphi$ и горизонталъным гомоморфизмом $\widehat{D} \varphi$, среди значений, определяемых горизонтальными гомоморфизмами вида $\widehat{D} \psi_{\varepsilon}$, необходимо, чтобы для $H_{m}^{S R}(x)$ из (17) выполнллосъ $H_{m}^{S R} \equiv 0, m=1, \ldots, \widetilde{n}$. 
Таким образом, уравнения $H_{m}^{S R} \equiv 0, m=1, \ldots, \widetilde{n}$, описывают классы минимальных поверхностей.

Пример 2. Если отображение определяется горизонтальным гомоморфизмом, не зависящим от $x \in \Omega$, то для него $H_{m}^{S R} \equiv 0, m=1, \ldots, \widetilde{n}$.

\section{ЛитеРАтУРА}

[1] Karmanova M., Vodopyanov S. Geometry of Carnot-Carathéodory spaces, differentiability, coarea and area formulas, Anal. and Math. Phys., 233-335 (Birkhäuser, Basel, 2009).

[2] Карманова М.Б. Вариации отображений с неголономным образом и применения $x$ теории максимальных поверхностей, Докл. РАН 468 (3), 257-260 (2016).

[3] Карманова М.Б. Максималъные поверхности на плтимерных групповых структурах, Сиб. матем. журн. 59 (3), 561-579 (2018).

[4] Карманова М.Б. Графики липшицевых функиий и минимальные поверхности на группах Карно, Сиб. матем. журн. 53 (4), 839-861 (2012).

[5] Folland G.B., Stein E.M. Hardy spaces on homogeneous groups (Princeton Univ. Press, Princeton, 1982).

[6] Pansu P. Métriques de Carnot-Carathéodory et quasi-isométries des espaces symétriques de rang un, Ann. Math. 129 (1), 1-60 (1989).

[7] Vodopyanov S. Geometry of Carnot-Carathéodory spaces and differentiablity of mappings, The Interaction Anal. and Geometry, Contemp. Math., (Amer. Math. Soc., Providence, RI, 2007), 424, 247-301.

[8] Карманова М.Б. О полиномиалъной субримановой дифференцируемости некоторых гёлъдеровых отображений групп Карно, Сиб. матем. журн. 58 (2), 305-332 (2017).

[9] Карманова М.Б. Формулы площади для классов гёлъдеровых отображений групп Карно, Сиб. матем. журн. 58 (5), 1056-1079 (2017).

Мария Борисовна Карманова

Институт математики им. С.Л. Соболева

Сибирского отделения Российской академии наук,

пр. Академика Коптюга, д. 4, г. Новосибирск, 630090, Россия,

e-mail:maryka@math.nsc.ru, maryka84@gmail.com

\section{M.B. Karmanova}

\section{Minimal graph-surfaces on arbitrary two-step Carnot groups}

Abstract. We establish basic properties of minimal graph-surfaces constructed from classes of mappings defined on two-step Carnot groups. Research methods include solving of a specific question on correctness of the problem statement. A main result on necessary minimality conditions is formulated in terms of sub-Riemannian analog of mean curvature.

Keywords: two-step Carnot group, graph-mapping, minimal surface, mean curvature.

Maria Borisovna Karmanova

Sobolev Institute of Mathematics,

Siberian Branch of Russian Academy of Sciences,

4 Acad. Koptyug Ave., Novosibirsk, 630090 Russia,

e-mail:maryka@math.nsc.ru, maryka84@gmail.com 